\title{
Mechanism of electron localization in a quantum wire
}

\author{
B S Shchamkhalova, V A Sablikov \\ Kotel'nikov Institute of Radioengineering and Electronics, Russian Academy of \\ Sciences, Fryazino, Moscow District, 141190, Russia \\ E-mail: bagun@ire216.msk.su
}

\begin{abstract}
.
We show that quasi-bound electron states are formed in a quantum wire as a result of electron backscattering in the transition regions between the wire and the electron reservoirs, to which the wire is coupled. The backscattering mechanism is caused by electron density oscillations arising even in smooth transitions due to the reflection of electrons not transmitting through the wire. The quasi-bound states reveal themselves in resonances of the electron transmission probability through the wire. The calculations were carried out within the Hartree-Fock approximation using quasiclassic wavefunctions.
\end{abstract}


Quantum point contacts (QPCs) and quantum wires (QWs) have attracted much interest as model systems for studying effects of an electron-electron interaction in onedimensional (1D) systems. The conductance of these devices is known [1, 2] to be quantized according to a universal law, $G=2 n e^{2} / h$, where $n=1,2,3 \ldots$, which was successfully explained within the model of noninteracting electrons [3, 4]. However, recent experiments have revealed a lot of other features of the conductance which are yet unexplained. Widely discussed is the 0.7 anomaly [5]. Besides, such observations as strongly nonlinear conductance at low applied voltage [6], additional plateaulike features [7] and even resonances in the differential conductance [7, 8, 9, 10] are also of interest. It is obvious that these features are caused by the electron-electron interaction, but only electron-electron interaction in the 1D system fails to explain them. Of no less importance is the fact that a $1 \mathrm{D}$ wire is coupled to $2 \mathrm{D}$ electron reservoirs and hence there are transition regions between the 1D QW and the 2D reservoirs (1D-2D junctions). The most puzzling result found recently is the electron localization in the QPC, which was first brought to light from the studies of the 0.7 plateaulike feature in short QWs. Cronenwett et al 11 related this feature to the Kondo effect caused by the electron spin localization in the QW. The most convincing evidence of the electron localization was provided by the momentum-resolved tunnelling experiments of Auslaender et al [12]. Peaks and kinks in conductance dependences on the gate voltage observed on the devices with high electron mobility [8, 7, 9] implicitly also points to the presence of quasi-bound electron states. The problem is that in all the experiments the electrons are localized over the barrier formed by the electrodes, the potential of which varies smoothly on the electron wavelength scale. The localization mechanism remains unclear, but it is widely believed that the existence of the quasi-bound states in the QW allows one to interpret the plateualike features and the resonances of the conductance. In [13 the quasi-bound states were related to local broadening of the QW and formation of a potential well. To justify the formation of the quasi-bound states, Hirose et al [14] calculated electron density distribution in QPCs with geometric lengths of the order of the Fermi wavelength. However in the experiments the geometric sizes exceed the Fermi wavelength noticeably, the barrier induced by the gates is rather smooth and the over-barrier reflection is negligible. Recently, Rejec and Meir [15] have demonstrated the presence of quasi-bound states in the QPC by calculations based on spin-density functional theory, but the underlying physical mechanism remains unknown.

Such a mechanism was suggested in [16]. The localization is a result of an intersubband electron interaction in the $1 \mathrm{D}-2 \mathrm{D}$ transition regions. The interaction is caused by the Friedel oscillations of the density of higher subband electrons, which do not pass through the transition regions, and are reflected. The electrons of an open subband are backscattered by these oscillations. Since the backscattering occurs in two opposed sides of the QW, quasi-bound states are formed. When studying the backscattering in 1D-2D junctions the problem is the complicated structure of the electron density distribution along the QW. Besides a smooth component of the electron density there is an oscillating one, and it is just this component that gives rise to the 

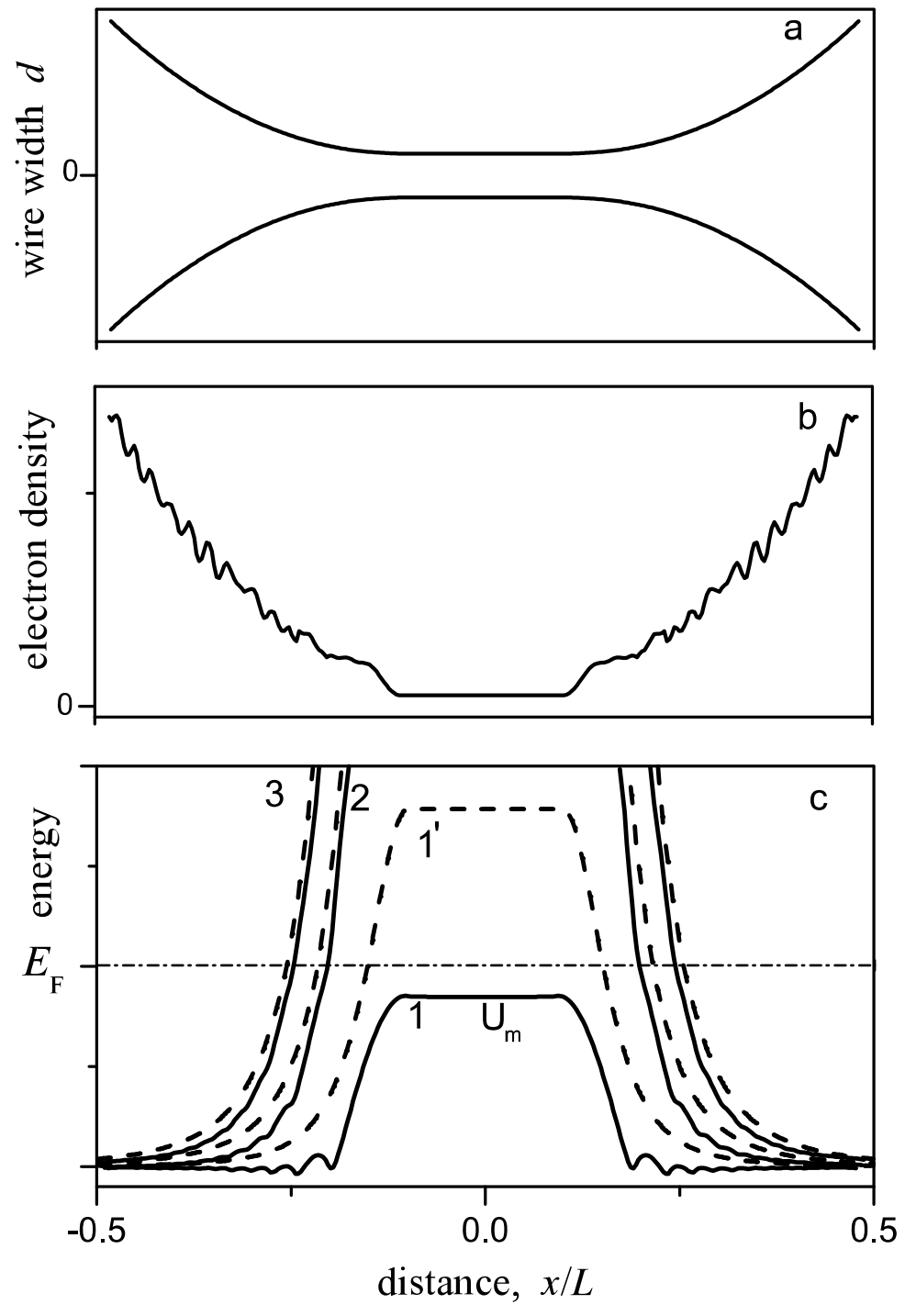

Figure 1. (a) Sketch of the device. (b) Effective 1D electron density. (c) Potential landscape and subband energies. Solid lines - selfconsistent relief $U(x)$ of the first (1) and higher $(2,3)$ subband bottoms; dashed lines - transverse quantization energies of the first (1') and higher subbands.

electron backscattering. An analytic theory of scattering by the Friedel oscillations in the outside of the junction (the far zone), where the wave vector of the oscillations is close to $2 k_{F}$ and the electrons with the Fermi energy are resonantly scattered, was developed in [16]. But the scattering by nonperiodic oscillations of the electron density inside the transition region (the near zone) may be also important, because the oscillation amplitude is larger there. In this paper the electron backscattering in the smooth 1D-2D junctions is studied within the Hartree-Fock approximation taking into account both near and far zones. The backscattering in the near zone turns out to contribute essentially to the total effect. It is found that quasi-bound states are formed in the QW giving rise to the transmission resonances.

Consider a QW in the form of a strip connecting 2D electron reservoirs with a given 
electrochemical potential $E_{F}$. The strip width, $d(x)$, varies as follows:

$$
d(x)= \begin{cases}d=\mathrm{const}, & |x|<a \\ d\left[1+(|x|-a)^{2} / R^{2}\right], & |x|>a,\end{cases}
$$

where the broadening radius $R$ considerably exceeds both $d$ and $k_{F}^{-1}\left(k_{F}\right.$ is the Fermi wave vector in reservoirs). For simplicity, we assume that only the first subband is open. The electrons in the higher subbands are reflected in the 1D-2D junctions.

The backscattering of electrons incident on the QW is calculated in a way similar to that of [16] with two essential additions which are required to include the scattering process in the near zone. First, in addition to the Friedel oscillations produced by closed subbands we take into account the electron density oscillations produced by the first subband electrons with energy below the potential landscape maximum in the QW, $U_{m}$, (figure 1(c)). These electrons are reflected from the barrier giving rise to electron density oscillations with the wave vector $\sim 2 \sqrt{2 m U_{m}} / \hbar$, which is close to $2 k_{F}$. Hence, these oscillations can noticeably contribute to the backscattering of the electrons passing through the QW. The second addition is the selfconsistent calculation of the smooth components of the potential and the electron density distribution in the QW. The potential landscape along the QW axis is formed by the transverse quantization energy and the smooth component of the Hartree potential. This is important because in the near zone the potential landscape considerably differs from the transverse quantization energy, as figure 1(c) shows.

The calculations are carried out in the following way. First, one-particle wave functions are written in the adiabatic approximation as a product of transverse and longitudinal wave functions

$$
\Psi_{n}\left(\vec{r}_{\perp}, x\right)=\phi_{n x}\left(\vec{r}_{\perp}\right) \psi_{n}(x) .
$$

where $n=1,2, \ldots$ is the subband number, $\phi_{n x}\left(\vec{r}_{\perp}\right)$ is a transverse wavefunction corresponding to transverse quantization energy $E_{n}(x)$. Second, effectively $1 \mathrm{D}$ equations are obtained for the longitudinal wave functions $\psi_{n}(x)$ by averaging the Hartee-Fock equations over transverse coordinates with the weight $\phi_{n x}^{*}\left(\vec{r}_{\perp}\right)$. As a result one obtains 1D Schrödinger equations with effective 1D Hartree and exchange terms. Third, these equations are solved selfconsistently using perturbation theory. To zero order in the interaction, the wave functions are written in the quasi-classic approximation. At this stage the problem is solved numerically using the iteration procedure developed in [17]. The electron scattering is calculated in the first Bohrn approximation. The quasiclassic approximation is justified if the local wavelength of an electron on the Fermi level is smaller than the characteristic spatial scale of the potential [18. The adiabatic approximation and its application to similar structures were considered in detail by Glazman et al [3, 19]

Thus, zero-order wave functions for the closed states are

$$
\psi_{n, k}(x)=2 \sqrt{\frac{k}{k_{n}(x)}} \cos \left[\int_{a_{n}}^{x} d x^{\prime} k_{n}\left(x^{\prime}\right)-\frac{\pi}{4}\right],
$$



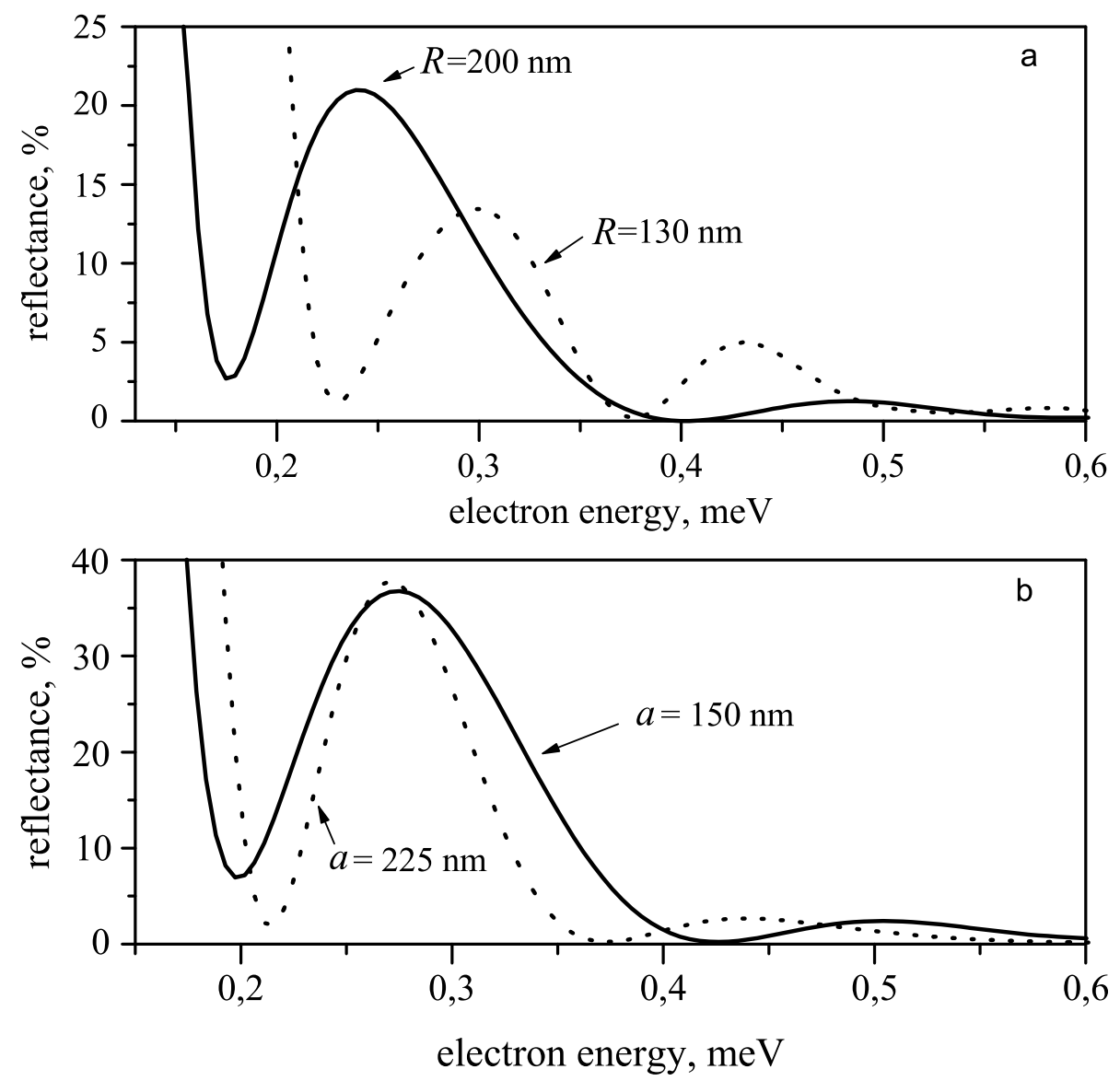

Figure 2. Reflection coefficient for different geometric sizes: (a) the widening radius $R$ is varied at $a=150 \mathrm{~nm}, D=600 \AA, E_{F}=9 \mathrm{meV}$; (b) the length $2 a$ of uniform part is varied at $R=135 \mathrm{~nm}, D=900 \AA, E_{F}=9 \mathrm{meV}$. For $a=150 \mathrm{~nm}$ (solid line), transmission resonances at energies 0.2 and $0.42 \mathrm{meV}$ are caused by second and third quasi-bound states in the QW. For $a=225 \mathrm{~nm}$ (dotted line) the transmission resonances correspond to third and fourth states.

where $k_{n}(x)$ is the wave vector of the longitudinal motion, $k=\lim _{x \rightarrow \infty} k_{n}(x)$ and $a_{n}(k)$ is a turning point. For electrons of the first subband with energy higher than the potential landscape maximum the zero-order wavefunction is

$$
\psi_{1, k}(x)=\sqrt{\frac{k}{k_{1}(x)}} \exp \left[i \int_{0}^{x} d x^{\prime} k_{n}\left(x^{\prime}\right)\right] .
$$

The electron density in each subband, having been found using equations (21) and (3)), depends on the electrochemical potential in the reservoirs $E_{F}$ and the effective potential $U^{n}(x)$ in the device. The density is a sum of two components: one is oscillating and the other varies smoothly on the electron wavelength scale. Accordingly, the potential acting on the electrons also has two similar components. A smooth component of the potential is calculated selfconsistently with the electron density using the technique developed in [17. As a result, the potential profile $U^{n}(x)$ (see Fig.11) is obtained for each subband and used to calculate the wave vectors $k_{n}(x)$ in equations (21) and (3), $k_{n}(x)=\sqrt{k^{2}-2 m U^{n}(x) / h^{2}}$. A rapidly oscillating component of the 
potential is considered as a perturbation, which scatters an electron from a state $|n, k\rangle$ to $\left|m, k^{\prime}\right\rangle$. The scattering potential contains the Hartree and exchange components: $\hat{V}=V^{H}+\hat{V}^{e x c}[16]$. The Hartree potential acting on the first subband electrons is

$$
V^{H}(x)=\sum_{l} \int d x^{\prime} V_{1, l}^{H}\left(x, x^{\prime}\right) \rho_{l}\left(x^{\prime}\right)-\int d x^{\prime} V_{1,0}^{H}\left(x, x^{\prime}\right) \rho_{0}\left(x^{\prime}\right),
$$

where $\rho_{l}(x)$ is the electron density in the lth subband, $\rho_{0}(x)$ is the background charge density and

$$
\begin{gathered}
V_{n l}\left(x, x^{\prime}\right)=\int V\left(\vec{r}, \vec{r}^{\prime}\right)\left|\phi_{n x}\left(\vec{r}_{\perp}\right)\right|^{2}\left|\phi_{l x}\left(\vec{r}_{\perp}\right)\right|^{2} d \vec{r}_{\perp} d \vec{r}_{\perp}^{\prime} \\
V_{n, 0}\left(x, x^{\prime}\right)=\int V\left(\vec{r}, \vec{r}^{\prime}\right)\left|\phi_{n x}\left(\vec{r}_{\perp}\right)\right|^{2} d \vec{r}_{\perp} d{\overrightarrow{r^{\prime}}}_{\perp} .
\end{gathered}
$$

The exchange interaction is described by an operator, which has following form for the first subband electrons

$$
\hat{V}^{e x c} \psi_{1, k}(x)=-\sum_{l} \int d x^{\prime} V_{1, l}^{e x c}\left(x, x^{\prime}\right) \rho_{l}\left(x, x^{\prime}\right) \psi_{1, k}\left(x^{\prime}\right)
$$

where $\rho_{l}\left(x, x^{\prime}\right)$ is the density matrix and

$$
V_{n, m}^{e x c}\left(x, x^{\prime}\right)=\int d \vec{r}_{\perp} d{\overrightarrow{r^{\prime}}}_{\perp} V\left(\mathbf{r}, \mathbf{r}^{\prime}\right) \phi_{n x}\left(\vec{r}_{\perp}\right) \phi_{m x}\left(\vec{r}_{\perp}\right) \phi_{n x}\left(\vec{r}_{\perp}\right) \phi_{m x}\left(\vec{r}_{\perp}\right) .
$$

Here $V\left(\mathbf{r}, \mathbf{r}^{\prime}\right)$ is the pair interaction potential. The screening of the Coulomb interaction is taken into account similarly to [17], assuming that the screening is produced by a conducting plane (gate) situated over the device at a distance $D$ or/and by the reservoirs, deep inside which the potential is fixed. In specific calculations the potential is taken to be constant at a distance $\pm L / 2$ from the center of the QW. The reflection amplitude for electrons in the open subband (i.e.,for the $(1, k) \rightarrow(1,-k)$ transition) is calculated in the Born approximation

$$
r_{k}=\frac{m}{i \hbar^{2}} \int d x \psi_{1, k}^{*} \hat{V} \psi_{1, k}
$$

The main results obtained are shown in figures 2,3], where the reflection coefficient $|r|^{2}$ of the electrons incident on the QW is shown as a function of the electron energy measured from the potential landscape maximum for a variety of the device parameters. One sees the resonant behavior of the electron reflection. At some energies the reflectance strongly diminishes and correspondingly the transmission resonantly increases. Calculations show that the resonance energies are mainly determined by the geometric sizes of the device: the length of the uniform part $2 a$ and the broadening radius $R$. This is demonstrated in figure 2, The screening effect on the reflectance is demonstrated by figure 3 where the reflectance spectra are shown for a variety of the distances $D$ between the QW and the screening electrode. This distance affects both the width of the transmission resonances (the width of the resonance decreases with $D)$ and the reflection coefficient at energies between the resonances $\left(|r|^{2}\right.$ increases with $D$ ), the resonance energies being weakly dependent on $D$. Similarly, the Fermi 


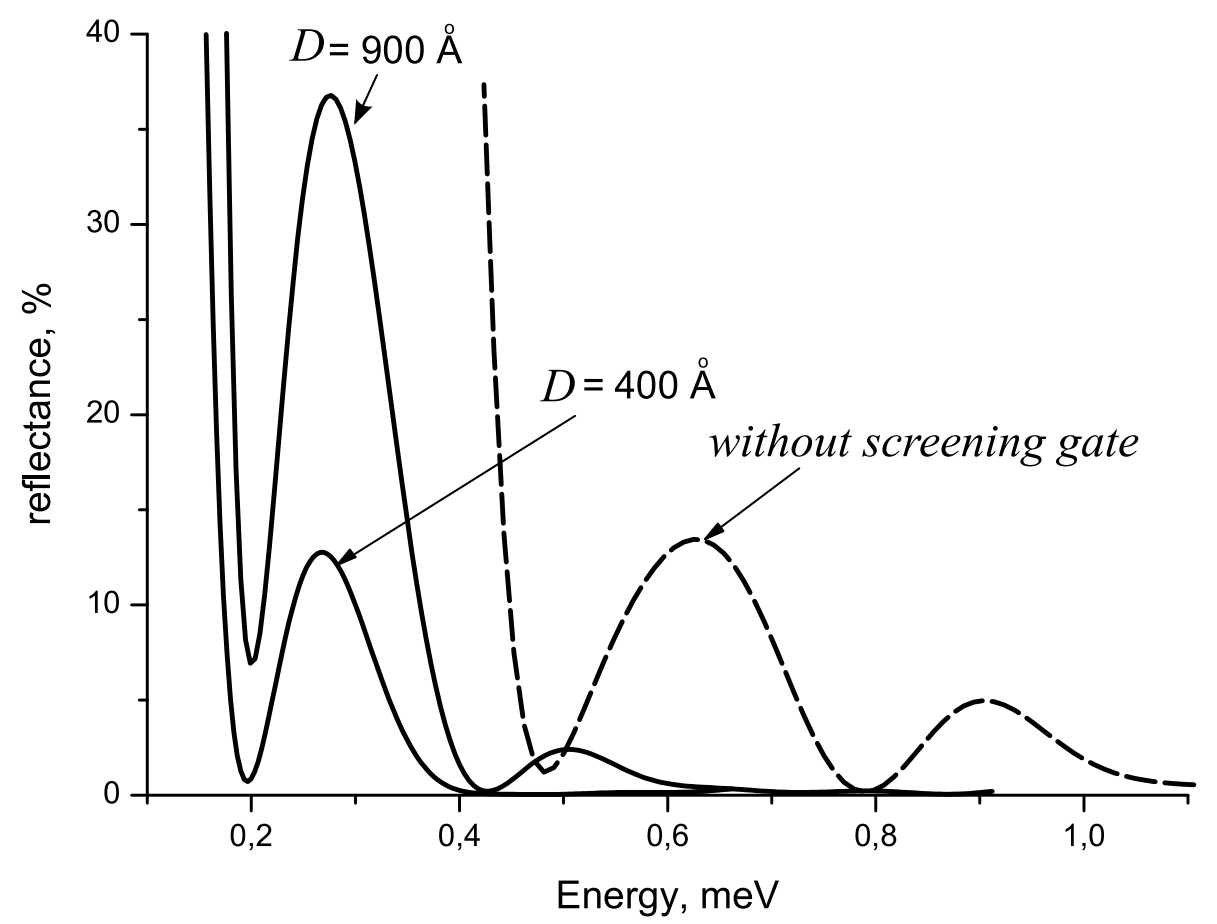

Figure 3. Reflection coefficient for different distance to the screening gate $D$. The parameters used are $a=150 \mathrm{~nm} ; R=135 \mathrm{~nm} ; L=1.5 \mu \mathrm{m} ; E_{F}=9 \mathrm{meV}$.

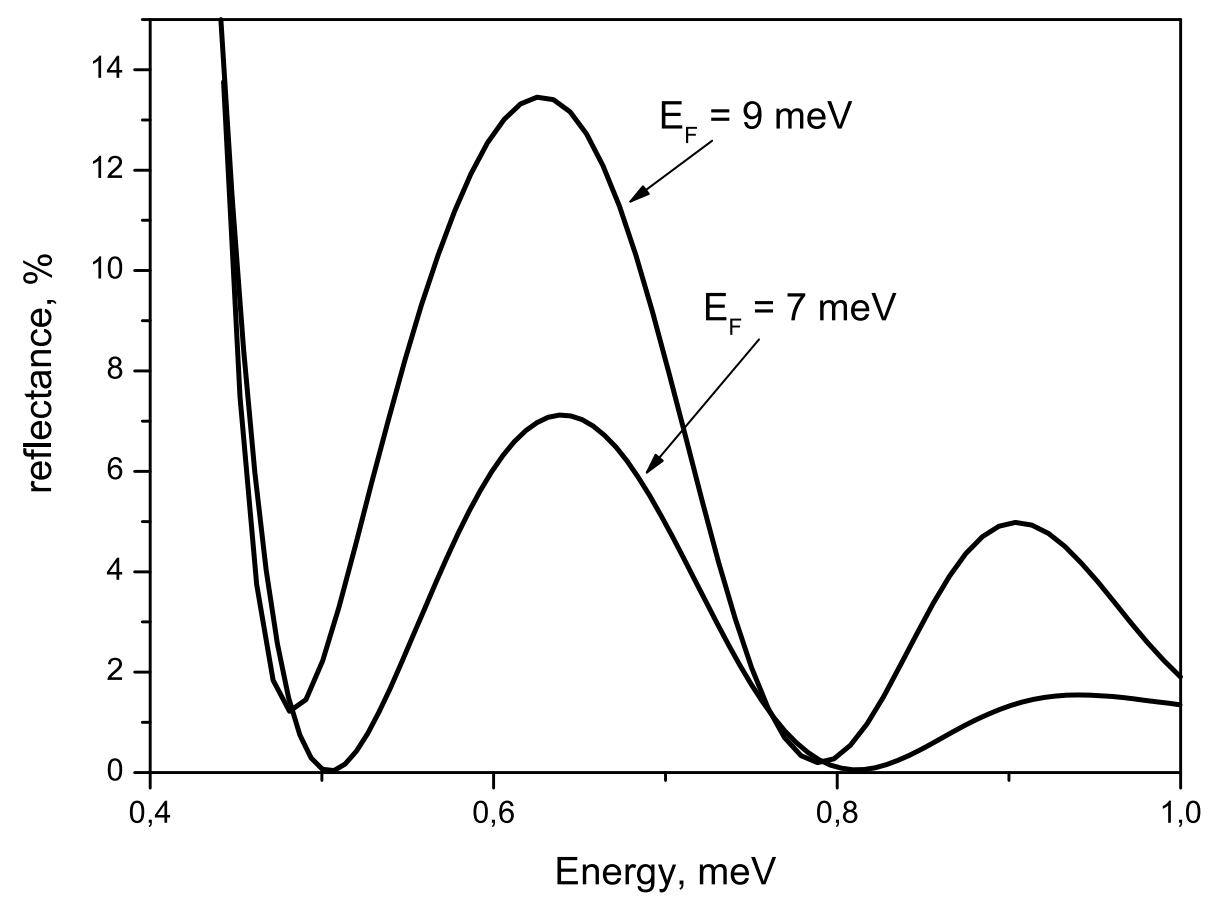

Figure 4. Reflection coefficient for different Fermi energies $E_{F}$ in reservoirs. The parameters used are $a=150 \mathrm{~nm} ; R=135 \mathrm{~nm} ; L=1.5 \mu \mathrm{m}$; screening gate is absent. 
level in the reservoirs weakly affects the position of the resonance, while $|r|^{2}$ is affected noticeably (figure 4).

The reflection resonances clearly point to the presence of quasi-bound states located in the region of the potential landscape maximum. The spectrum of the quasi-bound states and the energy dependence of the backscattering may be described rather well by a simple model. The electron scattering in 1D-2D junctions may be imagined as scattering by two $\delta$-like barriers located symmetrically at a distance $l$ from the QW center. The scattering potential is $W(x)=\Omega \delta(x \pm l)$. Here $l$ and $\Omega$ are fitting parameters. The wave vector $K$, for which the backscattering vanishes, is defined by the equation

$$
\tan (2 K l)=-\frac{2 K \hbar^{2}}{2 m \Omega}
$$

Using the ratio of energies of the sequential resonances in our numerical results we can define the serial numbers of the resonances. Then choosing the distance $l$ properly we can fit the resonance energies. The fitting leads to a simple equation:

$$
2 l=2 a+\gamma R
$$

where $\gamma$ is a parameter $(\gamma \simeq 0.5)$, which only slightly depends on the device geometry and the Fermi level in the reservoirs. The variation of the distance $D$ and the background positive charge density affect the power $\Omega$ of the effective scattering potential, which affects on the resonance energies slightly.

Thus, we have shown that the interaction between the electrons of the different subbands in 1D-2D junctions essentially affects the electron transport in QPCs and QWs. This interaction results in the transmission resonances which clearly evidence the formation of quasi-bound states in the region of the potential landscape maximum.

\section{Acknowledgments}

The work was supported by the Russian Foundation for Basic Research (project no. 0502-16854) and by Russian Academy of Sciences (programs "Quantum Nanostructures" and "Strongly Correlated Electrons in Semiconductors, Metals, Superconductors, and Magnetic Materials"), and the Ministry of Education and Science of RF.

\section{References}

[1] van Wees B J, van Houten H, Beenakker C W J, Williamson J G, Kouwenhoven L P, van der Marel D and Foxon C T 1988 Phys. Rev. Lett. 60, 848

[2] Wharam D A, Thornton T J, Newbury R, Pepper M, Ahmed H, Frost J T, Hasko D G, Peacock D C, Ritchie DE and Jones G A C 1988 J. Phys. C: Solid. St. Phys, 21 L209

[3] Glazman L I, Lesovik G B , Khmelnitskii DE and Shekhter R I 1988 JETP Lett. 48, 238

[4] Buttiker M 1990 Phys. Rev. B 41, 7906

[5] Thomas K J, Nicholls J T, Simmons MY, Simmons M Y, Pepper M, Mace D R and Ritchie D E 1996 Phys. Rev. Lett. 77, 135

[6] de Picciotto R, Pfeffer L N, Baldwin R W and West KW 2004 Phys. Rev. Lett. 92, 036805 
[7] Reilly D J, 2005 Phys. Rev. B 72, 033309

[8] Morimoto T, Ytnmi M, Naito R, Tsubaki K, Aoki N, Bird J P and Ochiai Y 2006 Phys. Rev. Lett. 97, 096801

[9] Shailos A, Ashok A, Bird J P, Akis R, Ferry D K, Goodnick S V, Lilly M P, Reno J L and Simmons J A 2006 J. Phys.: Cond. Matter, 181715

[10] Biercuk M J, Mason N, Martin J, Yacoby A and C. M. Marcus 2005, Phys. Rev. Lett. 94, 026801

[11] Cronenwett SM, Lynch HJ, Goldhaber-Gordon D, Kouwenhoven L P,Marcus C M, Hirose K, Wingreen N S and Umansky V 2002 Phys. Rev. Lett. 88, 226805

[12] Auslaender O M, Steinberg H, Yacoby A, Tserkovnyak Y, Halperin B I, Baldwin K W, Pfeiffer L N and West K W 2005 Science 308, 88

[13] Rejec T, Ramsak A and Jefferson J H, 2003 Phys. Rev. B 67, 075311

[14] Meir Y, Hirose K and Wingreen NS 2002 Phys. Rev. Lett. 89, 196802 ; Hirose K, Meir Y and Wingreen N S, 2003 Phys. Rev. Lett. 90, 026804

[15] Rejec T and Meir Y 2006 Nature 442, 900

[16] Sablikov V A 2006 JETP Lett, 84, 404; cond-mat/0611619

[17] Shchamkhalova B S and Sablikov V A 2005 Physica E 27, 51

[18] Zel'dovich Ya B and Rabinovich EM 1959 JETP 37, 1296

[19] Glazman L I and Jonson M, 1991 Phys. Rev. B 44, 3810 